\title{
Effect of a patient decision aid (PDA) for type 2 diabetes on knowledge, decisional self-efficacy, and decisional conflict
}

\author{
Robert. A. Bailey ${ }^{1}$, Michael Pfeifer ${ }^{1}$, Alicia C. Shillington ${ }^{2 *}$, Qing Harshaw ${ }^{2}$, Martha M. Funnell ${ }^{3}$,
} Jeffrey VanWingen ${ }^{4}$ and Nanada Col $^{5}$

\begin{abstract}
Background: Patients with type 2 diabetes (T2DM) often have poor glycemic control on first-line pharmacologic therapy and require treatment intensification. Intensification decisions can be difficult because of many available options and their many benefits and risks. The American Diabetes Association recommends patient-centered, evidence-based tools supporting shared decision-making between patients and clinicians. We developed a patient decision aid (PDA) targeting decisions about treatment intensification for T2DM. Our objective was to determine the effectiveness of this PDA for patients with T2DM on metformin who require treatment intensification.
\end{abstract}

Methods: This study was a pragmatic randomized controlled trial conducted in 27 US primary care and endocrinology clinics. Subjects were English-speaking adults with T2DM receiving metformin with persistent hyperglycemia who were recommended to consider medication intensification. Subjects were randomized to receive either the PDA or usual care (UC). Main outcome measures were change in knowledge, decisional self-efficacy, and decisional conflict.

Results: Of 225 subjects enrolled, 114 were randomized to the PDA and 111 to UC. Mean [SD] age was 52 [1] years, time since T2DM diagnosis was 6 [+/-6] years, $45.3 \%$ were male, and most (55.5\%) were non-Caucasian. Compared to UC, PDA users had significantly larger knowledge gains (35.0\% [22.3] vs $9.9 \%[22.2] ; P<0.0001)$ and larger improvements in self-efficacy (3.7 [16.7] vs-3.9 [19.2]; $P<0.0001)$ and decisional conflict $(-22.2$ [20.6] vs-7.5 [16.6]; $P<0.0001)$.

Conclusions: The PDA resulted in substantial and significant improvements in knowledge, decisional conflict and decisional self-efficacy. Decisional conflict scores after PDA use were within the range that correlates with effective decision-making. This PDA has the potential to facilitate shared-decision-making for patients with T2DM.

Trial registration: NCT02110979

Keywords: Type 2 diabetes mellitus, Antihyperglycemic medication, Shared decision-making, Patient decision aid, Decisional conflict, Decision self-efficacy

\section{Background}

Patients with type 2 diabetes mellitus (T2DM) with persistent hyperglycemia are at high risk of developing disease-related complications $[2,3]$. Choosing therapy for patients when first-line therapy with metformin is no longer effective is complex and involves difficult tradeoffs. Therapeutic options include adding an additional oral or injectable agent, including insulin [4]. A variety of antihyperglycemic medications are now available, each

* Correspondence: alicia.shillington@epi-q.com

2EPI-Q Inc, 1315 W 22nd Street, Suite 410, Oakbrook, IL 60523, USA

Full list of author information is available at the end of the article differing in important dimensions (e.g., effectiveness, side-effects, hypoglycemia risk, cost, impact on weight, and contraindications). There is no single best treatment appropriate for all patients, but rather the best treatment depends on what is important to each patient, their treatment goals, their underlying disease, and their comorbidities. Patients with diabetes often have inadequate information about their disease, their treatment options, and the consequences of their treatment decision, which can interfere with informed decision-making $[5,6]$. Inadequate knowledge about medication and self-care in patients with diabetes negatively impacts self-efficacy, the 
belief that one is informed, supported, capable of taking steps and making decisions to improve health $[7,8]$. Low self-efficacy is associated with lack of follow through on healthy intentions and decisions [9]. Inadequate knowledge and support also increase the likelihood of decisional conflict which is manifested by delays in decision-making, vacillating between choices, questioning values and tension [10]. Improving knowledge can promote diabetes self-efficacy, reduce decisional conflict, improve selfmanagement behaviors $[1,11,12]$, improve medication adherence [13, 14] and glycemic control [15-17], which should lead to improved clinical outcomes.

The American Diabetes Association (ADA) [18], and other medical organizations [19-22] recommend a patientcentered approach and patient engagement, including shared-decision making, to choosing among antihyperglycemic medications. They encourage clinicians to ensure that patients understand the decision and consider the risks and benefits involved in intensifying treatment (including the risk of hypoglycemia) [23, 24] and consider patient preferences. Helping patients make decisions aligned with their personal values is is integral to patient-centered care, an Institute of Medicine (IOM) mandate [25]. Yet implementing shared decision-making recommendations as a component of routine clinical care has been limited because of practice and health system barriers (e.g., limited time) and patient barriers (e.g., health literacy) [26]. Adoption of these recommendations will likely require targeted decision support tools to overcome these barriers.

Patient decision aids (PDAs) are educational tools designed to help patients make treatment decisions in collaboration with their clinician and promote shared decision-making. The Cochrane Collaboration systematic review of PDAs found they consistently improved knowledge, helped subjects match their values to their choices, and reduced passivity in decision-making [27]. PDAs focusing on patients with T2DM addressed statin choice [28-31] goal-setting [32, 33] metabolic control [34], addition of insulin [35], and starting or changing statins or antihyperglycemic treatment [36]. These PDAs promoted patients' discussions of medications with their clinician, realistic expectations, autonomy, trust, engagement in decision-making, patient knowledge, risk perceptions, and documented goals, but none addressed decisional self-efficacy. Self-efficacy has been shown to be an important mediator of health decisions and health behaviors [37]. Additionally, none of these PDAs targeted the important decision about further treatment options that confronts the many patients with T2DM for whom first-line therapy with metformin is no longer effective and who need to consider further treatment options. This study was conducted in 27 non-academic sites across the country, giving much-needed evidence about the use of PDAs is real world environments.
The objective of this study was to determine the impact of a PDA for decisions about antihyperglycemic medications on key elements of shared decision-making (knowledge, self-efficacy, and decisional conflict) in a pragmatic randomized controlled trial involving a diverse national sample of subjects with T2DM during the course of routine clinical care.

\section{Methods}

We conducted a multicenter randomized controlled pragmatic trial (Clinical Trials.gov Identifier: NCT02110979).

\section{Setting and participants}

This trial was conducted in 27 primary care or endocrinology clinics throughout the US (Additional file 1). To improve the generalizability of the study, we restricted our national U.S. sample to non-academic clinical sites. Clinics were identified through a nationwide email list of providers maintained by the Primary Care Network $^{\mathrm{m}}$ (Springfield, $\mathrm{MO}$ ), supplemented by providers' emails obtained through a medical marketing database $\left(\mathrm{IMD}^{\circ}\right)$. Clinicians were invited to participate and were selected if they were primary care clinicians or endocrinologists treating a minimum of ten patients with T2DM each week, had access to electronic medical records and/or electronic laboratory data, had a nurse, diabetes educator or other clinical staff support to facilitate subject identification, and were not affiliated with an academic institution. Approximately 2000 emailed invitations were sent; 68 sites responded; 32 were selected and 27 enrolled at least one subject.

Subject inclusion criteria: Eligible subjects were Englishspeaking adults with T2DM who were advised by their clinician to consider additional antihyperglycemic medication to a metformin-containing regimen to improve glycemic control. Subjects were further required to have a valid email address, access to the internet via a personal computer, and the ability to complete surveys online.

Subject exclusion criteria: Excluded were pregnant women, clinical trial participants, subjects already taking two or more medications in addition to metformin, or those with a lifetime exposure to more than three antihyperglycemic agents.

Patient Consent Procedures: All study procedures were approved by the New England IRB. Patient recruitment was intended to simulate how the PDA would be used in the 'real world' after trial completion. Potential study subjects were identified using medical records based on recent laboratory testing assessing glycemic control and rosters of appointments. Potential subjects were referred to the principle investigator by their clinician. Subjects were emailed a link to the study website (maintained by Qualtrics ${ }^{\circ}$ ) which screened for eligibility, obtained online consent, and collected baseline data. Subjects were randomized to either the online intervention (PDA group) or 
usual care (UC group). UC subjects were directed to follow-up with their doctor at an upcoming appointment as they normally would. Usual care was selected as the control arm in order to understand the incremental benefit of the PDA beyond care typically received during a clinical consultation and to make findings relevant to clinicians considering incorporating PDAs into routine practice. Referring clinicians were blinded to group assignment, unless they were incidentally un-blinded by subjects during a clinical consultation subsequent to enrollment (e.g., subjects mentioning the PDA or its contents during an office visit). Consistent with an intention-to-treat (ITT) principle $[38,39]$, we attempted to minimize loss to followup by monitoring subjects and prompting non-responders or partial responders to complete the intervention and/or assessments through phone and email reminders.

\section{The PDA}

The interactive Diabetes Decision Aid for T2DM targeted decisions about antihyperglycemic medication intensification for subjects for whom first-line treatment with metformin is no longer effective. The evidence-based PDA was designed to help subjects understand T2DM, its natural history, and the full range of treatment options, including sulfonylureas, dipeptidyl peptidase-4 (DPP-4 s) inhibitors, thiazolidinediones (TZDs), sodium-glucose co-transporter two (SGLT-2) inhibitors, glucagon-like peptide-1 (GLP-1 s) agonists, and insulin. To make the information more salient to subjects, evidence about the risks and benefits of different treatment options were organized and presented according to key preference domains that were identified during the development process [40] and through two focus groups with affected subjects. These domains include: 1) expected degree of glycemic control, 2) impact on weight, 3) risk of hypoglycemia and other adverse events, 4) route of administration, 5) frequency of dosing and route of administration and blood glucose monitoring, and 6) cost of therapy. The PDA described and compared the potential benefits and risks of the treatments in each of these domains and helped subjects explore their preferences and values through values clarification exercises [41]. It included questions eliciting their long-term goals for therapy and their concerns about antihyperglycemic medications. A summary "fact sheet" compared the risks and benefits of each class of medication using voice-over descriptions and simple graphics. Potential risks and benefits were illustrated using color-coded pictographs with plain language summaries, presented in a balanced, unbiased manner (Fig. 1).

The PDA did not allow subjects to skip through content in order to ensure that all key content areas were reviewed. It did not discuss lifestyle interventions (e.g., smoking, diet and exercise) but it did enable subjects to request information on these topics. The PDA was developed in accordance with the International Patient Decision Aid Standards criteria [42] in collaboration with a multidisciplinary team with expertise in clinical medicine, shared decision-making, and patient education [38].

\section{Intervention protocol}

Prior to enrolling any subjects, clinicians and staff were encouraged to review the online PDA. Clinicians and clinic staff involved in identifying potential subjects participated in brief training sessions $(30-60 \mathrm{~min}$ ) on the use of the PDA and study procedures. Subjects randomized to the PDA group received computer-generated emails asking them to view information "recommended by their doctor" and a link to the PDA. Reminder messages were sent daily until the subject viewed the PDA in its entirety. Subjects who had not viewed the PDA within 5 days were contacted by phone or e-mail as a further reminder. The PDA took approximately $30 \mathrm{~min}$ to complete and subjects were encouraged to watch it as many times as they wanted and to make use of an online note-taking system to record questions or comments that could be shared with their doctor. Subjects were encouraged to view the PDA with others involved in the decision-making process.

\section{Outcomes and data collected}

Study data were collected at baseline and 4-6 weeks following enrollment into the study for both study groups. In the PDA group, a brief survey was distributed shortly after viewing the PDA (within approximately $24 \mathrm{~h}$ ) to assess opinions related to detail and length of content. A four to 6 weeks measurement time frame was selected in order to capture the impact of the PDA on knowledge gained, self- efficacy, and decisional conflict, outcomes which are best captured within the first month after exposure to the PDA.

Clinicians responded to similar questions. Subjects were paid $\$ 25$ at each data collection point.

\section{Baseline data}

All subject data was self-reported and included sociodemographics (age, gender, race/ethnicity, educational level, employment and insurance status), duration of T2DM, height and weight, and relevant co-existing conditions (Table 1). Subjects were asked about their stage of decision-making [43], (e.g., had they begun to think about the decision, had they already made a decision), their preference for making clinical decisions, (e.g., do they prefer to have their clinician make decisions, prefer shared-decision-making or prefer making decisions on their own) [44] and the relative importance of competing values related to domains of diabetes medication decisionmaking. Subject values assessed included the importance of: managing blood sugar to a goal; taking a medication 


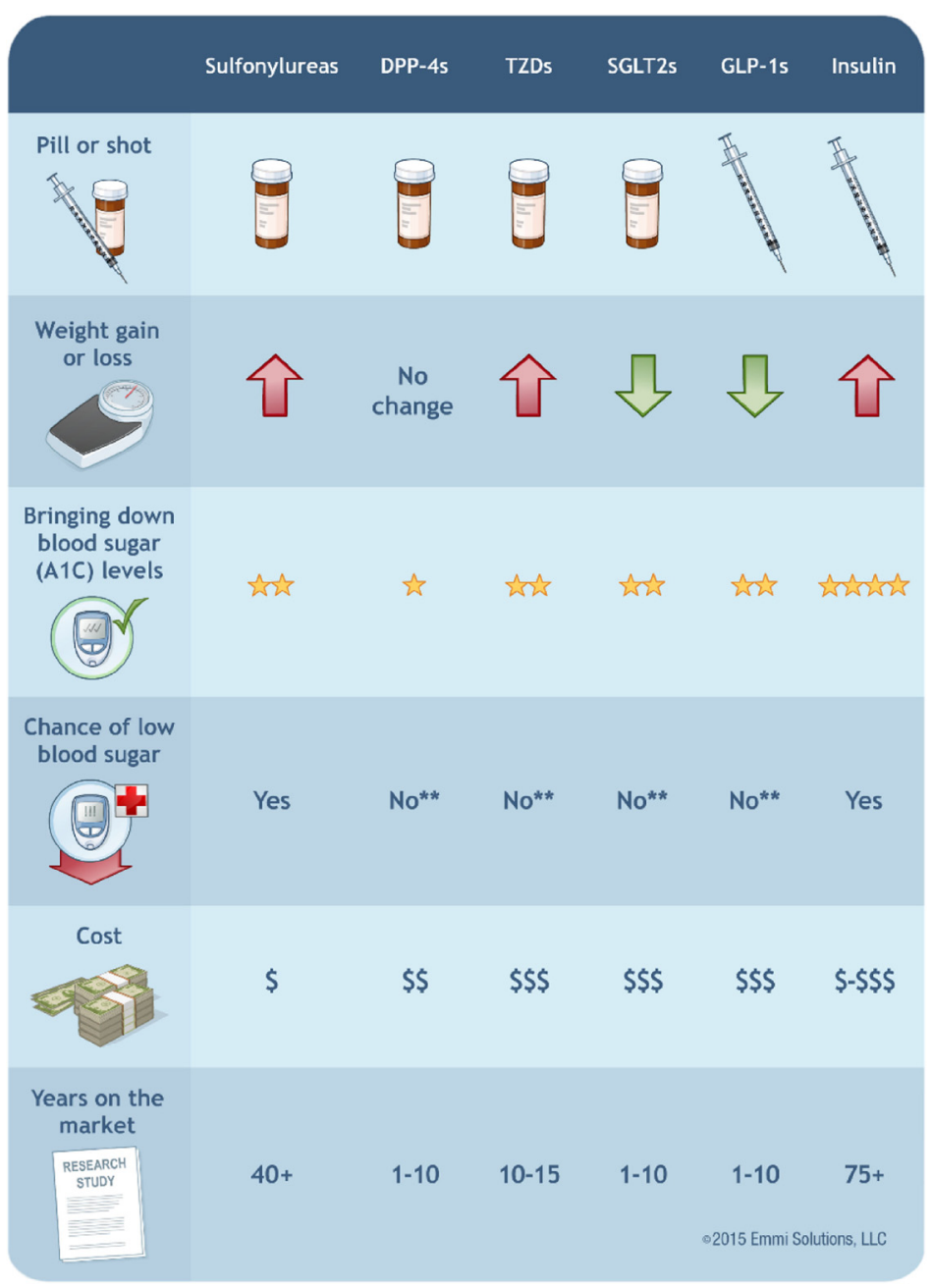

*DPP-4 - dipeptidyl peptidase-4 inhibitors, TZD - thiazolidinediones, SGLT-2 - ,

sodium-glucose co-transporter 2 inhibitors, GLP-1 -glucagon-like peptide-1agonists

Fig. 1 Type 2 Diabetes Medications Comparison Chart*. PDA "Fact Sheet"

that might help lose weight or not cause weight gain; avoiding hypoglycemia (a "low"); avoiding side-effects such as pancreatitis, fractures, urinary tract infections and yeast infection; treatment costs, avoiding injections; and dosing convenience (i.e., taking medication more than once a day). These were assessed using a scale from 0 to 10, with 0 representing not at all important, and ten representing extremely important.

\section{Primary outcome}

The primary outcome of this study was knowledge gained about medication, using a scale developed for the study to assess understanding of how different treatments differ in terms of their 1) impact on glycemic control (amount and durability), 2) impact on weight, 3) risk of hypoglycemia and other adverse events, 4) route of administration, 5) frequency of dose administration and blood glucose monitoring, and 6) financial costs. Knowledge was assessed by asking subjects to respond to 17 statements about antihyperglycemic medications that were either true or false (Additional file 1). To discourage guessing, we offered a "not sure" response option. We calculated the percentage of correct answers. We also asked subjects to rate their confidence in their responses using a ten point Likert scale (from 1, "I am certain this is incorrect" to ten, "I am certain this is correct", with five corresponding to "not sure"). All questions were pretested on ten subjects with T2DM and revised accordingly. The pretest suggested uniform comprehension of all but one item, which was subsequently reworded. A composite "perceived knowledge confidence" score was summed and normalized to a $0-100$ scale. Knowledge gained was calculated by subtracting baseline from follow-up scores. 
Table 1 Baseline characteristics

\begin{tabular}{|c|c|c|c|}
\hline & $\operatorname{PDA}(n=114)$ & Usual Care $(n=111)$ & $p$-value $e^{a}$ \\
\hline Mean age (Mean, SD) & $53.0(13.8)$ & $51.6(11.5)$ & 0.4121 \\
\hline Male $(n, \%)$ & $52(45.6)$ & $50(45.1)$ & 0.9317 \\
\hline \multicolumn{4}{|l|}{ Race/Ethnicity (n, \%) } \\
\hline White/Caucasian & $53(46.5)$ & $48(43.2)$ & 0.6243 \\
\hline Black/African American & $32(28.1)$ & $31(27.9)$ & 0.9810 \\
\hline Hispanic & $14(12.3)$ & $15(13.5)$ & 0.8437 \\
\hline Asian/Pacific Islander & $5(4.4)$ & $9(8.1)$ & 0.2810 \\
\hline Native American or Alaska Native & $1(0.9)$ & $2(1.8)$ & 0.6183 \\
\hline Other or Multi-Racial & $9(7.9)$ & $6(5.4)$ & 0.4542 \\
\hline \multicolumn{4}{|l|}{ Education (n, \%) } \\
\hline Grade School & $2(1.8)$ & $1(0.9)$ & 0.5786 \\
\hline Some High School & $12(10.5)$ & $2(1.8)$ & 0.0068 \\
\hline High School Graduate & $29(25.4)$ & $29(26.1)$ & 0.9062 \\
\hline Some College & $30(26.3)$ & $44(39.6)$ & 0.0334 \\
\hline College Graduate & $26(22.8)$ & $24(21.6)$ & 0.8307 \\
\hline Graduate School & $15(13.2)$ & $11(9.9)$ & 0.4461 \\
\hline \multicolumn{4}{|l|}{ Employment (n, \%) } \\
\hline Full time & $50(43.9)$ & $60(54.1)$ & 0.3379 \\
\hline Part time & $11(9.6)$ & $7(6.3)$ & \\
\hline Unemployed & $15(13.2)$ & $11(9.9)$ & \\
\hline On Disability & $19(16.7)$ & $11(9.9)$ & \\
\hline Student & $2(1.8)$ & $2(1.8)$ & \\
\hline Homemaker & $6(5.3)$ & $3(2.7)$ & \\
\hline Other & $11(9.6)$ & $17(15.3)$ & \\
\hline \multicolumn{4}{|l|}{ Insurance status (n, \%) } \\
\hline PPO & $39(34.2)$ & $49(44.1)$ & 0.2585 \\
\hline $\mathrm{HMO}$ & $26(22.8)$ & $14(12.6)$ & \\
\hline Medicare & $19(16.7)$ & $22(19.8)$ & \\
\hline Medicaid & $16(14.0)$ & $10(9.0)$ & \\
\hline None & $6(5.3)$ & $6(5.4)$ & \\
\hline Other & $8(7.0)$ & $10(9.0)$ & \\
\hline Years since T2DM Diagnosis (Mean, SD) & $6.8(6.0)$ & $6.5(5.4)$ & 0.7192 \\
\hline BMI (Mean, SD) & $34.4(8.0)$ & $35.6(9.4)$ & 0.3152 \\
\hline Underweight $(<18.5)(n, \%)$ & 0 & 0 & 0.8106 \\
\hline Normal (18.5-24.99) & $13(11.4)$ & $11(9.9)$ & \\
\hline Overweight (25-29.99) & $22(19.3)$ & $21(18.9)$ & \\
\hline Obese class I (30-34.99) & $32(28.1)$ & $27(24.3)$ & \\
\hline Obese class II (35-39.99) & $15(13.1)$ & $21(18.9)$ & \\
\hline Obese class III (>40) & $32(28.1)$ & $31(27.9)$ & \\
\hline \multicolumn{4}{|l|}{ Comorbidities and Health Conditions $(n, \%)$} \\
\hline High Blood Pressure & $82(71.9)$ & $69(62.2)$ & 0.1190 \\
\hline Heart Disease & $10(8.8)$ & $9(8.1)$ & 0.8579 \\
\hline Stroke & $3(2.6)$ & $5(4.5)$ & 0.4951 \\
\hline High Cholesterol & $59(51.8)$ & $57(51.4)$ & 0.9518 \\
\hline
\end{tabular}


Table 1 Baseline characteristics (Continued)

\begin{tabular}{|c|c|c|c|}
\hline High Triglycerides & $23(20.2)$ & $27(24.3)$ & 0.4542 \\
\hline Gallbladder Disease & $8(7.0)$ & $5(4.5)$ & 0.4192 \\
\hline Liver Disease & $2(1.8)$ & $2(1.8)$ & 1.000 \\
\hline Kidney Disease or Renal Failure & $1(0.9)$ & $2(1.8)$ & 0.6183 \\
\hline Pancreatitis & $3(2.6)$ & $5(4.5)$ & 0.4951 \\
\hline Insulin Resistance & $5(4.4)$ & $7(6.3)$ & 0.5216 \\
\hline Osteoporosis & $3(2.6)$ & $2(1.8)$ & 1.000 \\
\hline Peripheral Vascular Disease & $5(4.4)$ & $5(4.5)$ & 1.000 \\
\hline Hypoglycemia & $3(2.6)$ & $5(4.5)$ & 0.4951 \\
\hline Ketoacidosis & 0 & $2(1.8)$ & 0.2423 \\
\hline Neuropathy & $12(10.5)$ & $9(8.1)$ & 0.5330 \\
\hline Yeast or Urinary Tract Infection & $22(19.3)$ & $11(9.9)$ & 0.0466 \\
\hline \multicolumn{4}{|c|}{ Baseline Knowledge, Decisional Conflict and Decision Self-efficacy } \\
\hline Knowledge (\% correct, mean, SD) & $20.4 \%(15.7)$ & $22.6 \%(16.9)$ & 0.3150 \\
\hline Knowledge confidence score (mean, SD) & $56.1(5.6)$ & $56.9(8.6)$ & 0.4035 \\
\hline Decisional Conflict Scale (DCS) (mean, SD) & $45.8(18.8)$ & $45.4(16.9)$ & 0.8559 \\
\hline Uncertainty subscale & $49.9(24.5)$ & $50.8(24.9)$ & 0.7915 \\
\hline Informed subscale & $56.9(23.7)$ & $58.5(24.2)$ & 0.6214 \\
\hline Support subscale & $36.6(19.7)$ & $37.2(18.4)$ & 0.8422 \\
\hline Values Clarity subscale & $48.6(23.2)$ & $45.1(22.9)$ & 0.2524 \\
\hline Effective Decision subscale & $39.1(19.5)$ & $37.8(16.5)$ & 0.5880 \\
\hline Decisional Self-Efficacy Scale (DSES) (mean, SD) & 85.9 (15.6) & $85.6(16.1)$ & 0.8738 \\
\hline
\end{tabular}

${ }^{a}$ numerical variables $t$-test; categorical variables: $\mathrm{X}^{2}$ or Fisher's exact test

\section{Secondary outcomes}

Secondary outcomes included changes in the Decision Self Efficacy Scale (DSES) and Decisional Conflict Scale (DCS). The DSES assesses subjects' self-confidence or belief in one's abilities in decision-making. In testing, the DSES internal reliability coefficient was .92 , and the scale significantly discriminated between subjects who did and did not make health-related decisions [45]. The DSES is summed and scored from 0 to 100 with 100 representing complete self-efficacy and 0 representing complete lack of self-efficacy. The DCS [46] measures overall uncertainty in making a health-related decision and includes subscales that address the factors that contribute to uncertainty (feeling uncertain, informed, clear about values, supported in effective decision-making). In validation testing, the test-retest reliability coefficient of the DCS was 0.81. Internal consistency coefficients ranged from 0.78 to 0.92 . The DCS discriminated significantly between those who had strong intentions for and ultimately acted upon decision making and those whose intentions were uncertain [10]. The DCS and subscales are scored from 0 to 100; lower scores correspond to less decisional conflict [47]. Changes in DSES and DCS were calculated by subtracting baseline from follow-up scores.

\section{Data analyses}

All subjects were analyzed in the arm to which they were randomized (ITT). Baseline demographic, past medical history and responses to baseline decisionrelated questionnaires were compared between treatment groups in univariate analyses to assess the success of randomization, using t-tests for continuous and $\mathrm{X}^{2}$ or Fisher's exact for categorical data. For the primary and secondary outcomes, unadjusted mean differences in knowledge, DSES and DCS scores were calculated by subtracting post from baseline scores and comparing those values using t-tests. Covariate adjusted analysis on primary and secondary outcome were conducted using generalized linear models (GLM). Dependent variables in the models were knowledge gained, knowledge confidence score, DCS and DSES. Covariates included in the models were those of theoretical interest to the investigators (e.g. stage of decision-making, race/ethnicity) and those relating to failed randomization, using a threshold of $\mathrm{p}<0.1$.

Adherence to the intervention group (viewing the PDA) was included in GLM models as a dichotomous variable (all or none), based upon tracking each participant's unique subject code for accessing the PDA. UC 
controls did not have access to PDA; crossovers from the control to intervention group were not possible.

We assessed level of missing data for our key constructs and outcomes. No variables had $>30 \%$ missing thus we imputed missing data using a monotone data Markov-Chain-Monte-Carlo (MCMC) method (SAS/ STAT procedure MIANALYZER) [48]. Analyses were computed using SAS (SAS Institute, Inc, Cary, NC).

\section{Data collection, quality and integrity}

The survey software system (Qualtrics ${ }^{\circ}$ ) that was used to enroll, screen, and implement surveys has a variety of features to ensure data integrity. The survey questionnaires could only be completed by the person to whom links were sent, IP addresses were independently assessed to verify no unauthorized access, and data were written directly to the secure Qualtrics ${ }^{\oplus}$ database, which could not reviewed or changed once submitted by subjects as complete. All staff analyzing data were blinded to treatment group assignment. Referring clinicians were blinded to group assignment, unless they were incidentally unblinded by subjects during a clinical consultation subsequent to enrollment (e.g., subjects mentioning the PDA or its contents during an office visit).

\section{Power analyses}

Our study was powered to measure the impact of the PDA on the primary outcome of knowledge, using a 2tailed test. In a Cochrane systematic review of 42 studies (10842 participants) measuring the impact of PDA vs usual care on knowledge the effect size was 13.34 [11.17, 15.51] [26]. Our study with a sample size of 110 in each of two arms was projected to have a $90 \%$ chance to detect a difference of 13.34 or greater in knowledge gained.

\section{Results}

We randomized 225 subjects into the study (Fig. 2), 114 to the PDA and 111 to usual care. All subjects were followed for approximately 6 weeks after randomization except for 20 who were lost to follow-up (PDA group, $n=$ 15; usual care group, $n=5$ ). Twenty seven (27) clinicians enrolled at least 1 subject, with 15 enrolling more than five subjects.

Baseline demographic and clinical characteristics are presented in Table 1. The mean age of all subjects was 52.3 [sd 12.7] years, the majority were female $(54.7 \%)$ and nonCaucasian (55.5 \%). Mean duration since T2DM diagnosis was 6.6 [sd 5.7] years. Baseline demographics and comorbidities were similar across both groups, with two exceptions. Fewer subjects in the PDA versus UC group reported having some college education ( $26.3 \%$ vs $39.6 \%$, respectively), and fewer had a history of a yeast or urinary tract infection (UTI). Most (57.3 \%) had not begun considering their choices for medications; most $(71.1 \%)$ preferred an active role in health care decision-making, either shared with their physician, or independently (Table 2).

Figure 3 compares the importance that participants placed on different outcomes affected by treatment ('subject values'). There were no differences between the study groups so both groups were combined. The most important values were a medication's ability to manage blood sugar to a goal, followed closely by avoidance of sideeffects. Least important were the convenience of dosing.

\section{Knowledge outcomes}

Subjects in the PDA group gained substantially more knowledge than those in the UC group at 6 week followup $(35.0 \%$ versus $9.9 \%$ improvement in scores, respectively, $p<0.0001$ ). (Table 3 ) This corresponds to subjects in the PDA group correctly answering nine of 17 knowledge questions regarding medication treatments. Knowledge confidence also improved substantially more in the PDA group versus UC (11.0 [sd 12.8] versus 1.6 [sd 8.9] respectively $(p<0.0001)$. In both groups, knowledge confidence score was correlated with knowledge, $r=0.476(p<0.001)$.

In multivariate analyses that adjusted for gender, educational level, race, stage of decision-making, importance of cost in making medication decisions, comorbidities, and other factors, PDA use (versus usual care) was associated with a greater likelihood of knowledge score gains, 26.6 percentage points $(p<0.0001)$ and a 10.1 points $(p<$ 0.0001) gain in knowledge confidence (Table 4).

\section{Decisional self efficacy and decisional conflict outcomes}

PDA users, as compared to UC, had substantially larger likelihood of improvements in both decisional selfefficacy (3.7 vs-3.9, respectively) and decisional conflict (-22.2 vs-7.5, respectively) within 6 weeks of enrollment, differences which were highly statistically significant $(p<.0001)$. The mean DCS score among PDA users at final follow-up was well under 25 (23.6 [sd 14.3]). Significant improvements in each of the decisional conflict scale sub-scores were observed (Table 3).

Multivariate analyses adjusting for educational, race, stage of decision-making, importance of cost in making medication decisions, previous yeast or urinary tract infections, and other factors found that PDA use was associated with a substantial likelihood of decline in decisional conflict $(-16.25(p<0.0001))$ and improvement in decisional self-efficacy ( $7.44[p=0.004]$ ) (Table 4).

Among those assigned to the intervention group, 95 (83.3\%) responded to a brief email survey shortly after viewing the PDA. 94 (98.9\%) reported the information was "just right" and "well balanced" and $90 \%$ that the information was sufficient to understand each class of antihyperglycemic agent's effectiveness and side effects. Twelve (12.6\%) found the length of the material "too long". Ten clinicians who enrolled a minimum of ten subjects were 


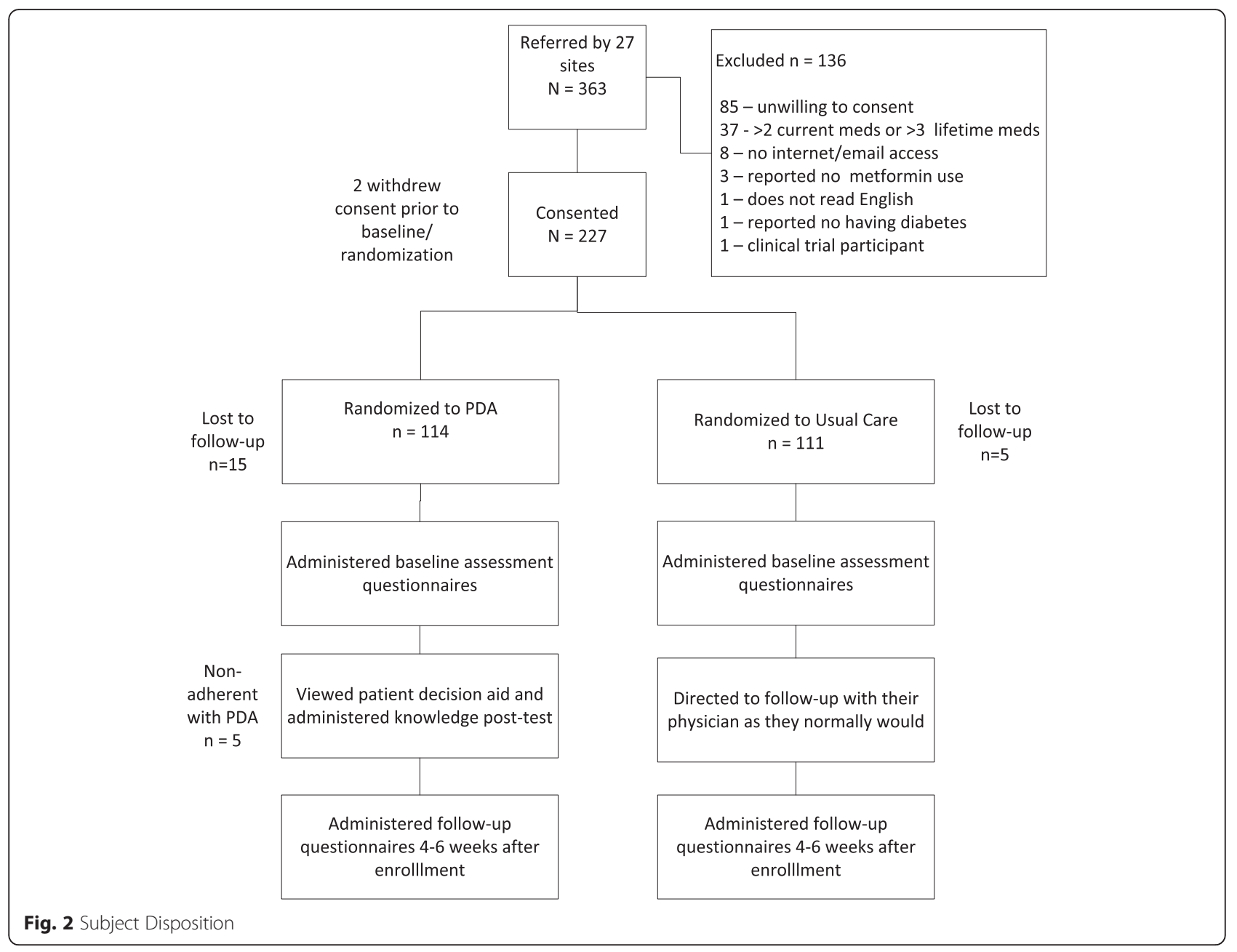

Table 2 Baseline decisional characteristics

\begin{tabular}{|c|c|c|c|}
\hline & PDA & Usual Care & $p$-value \\
\hline & $n=114$ & $n=111$ & \\
\hline Stage of Decision-making & $\mathrm{n}(\%)$ & $\mathrm{n}(\%)$ & \\
\hline Haven't begun to think about the choices & $37(32.5)$ & $31(27.9)$ & 0.4596 \\
\hline Haven't begun to think about the choices, but am interested in doing so & $30(26.3)$ & $31(27.9)$ & 0.7856 \\
\hline ....are considering the options now & $18(15.8)$ & $26(23.4)$ & 0.1489 \\
\hline ....are close to selecting an option & $3(2.6)$ & $1(0.9)$ & 0.6218 \\
\hline Have already made a decision, but am still willing to reconsider & $15(13.2)$ & $14(12.6)$ & 0.9029 \\
\hline Have already made a decision and am unlikely to change my mind & $11(9.7)$ & $8(7.2)$ & 0.5101 \\
\hline \multicolumn{4}{|l|}{ Control Preference } \\
\hline Doctor-oriented & $34(29.8)$ & $31(27.9)$ & 0.7537 \\
\hline Shared & $51(44.7)$ & $58(52.3)$ & 0.2594 \\
\hline Independent & $29(25.4)$ & $22(19.8)$ & 0.3142 \\
\hline
\end{tabular}

${ }^{a} X^{2}$ test or Fisher exact test 


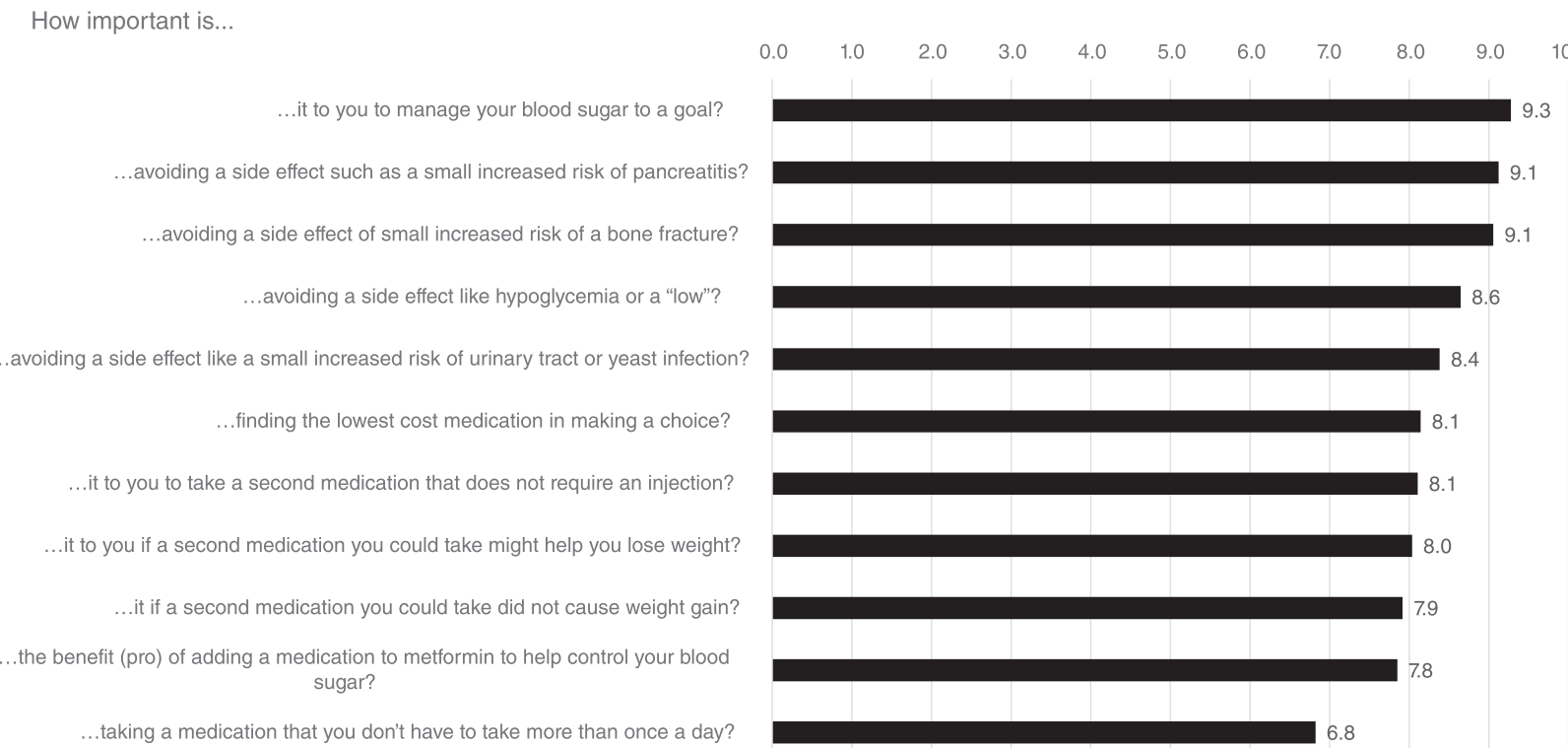

9.3 9.1

Fig. 3 Subject Values Scores At Baseline for Outcomes Related to Treatment

asked to re-review the PDA at the end of the study and answer (a) similar question. Of the nine who responded, six $(66.6 \%)$ endorsed the statement that the PDA "could help patients to fully understand the risks and benefits of treatment options for T2DM" either "a great deal" or "quite a bit", with the remainder (33.3\%) responding "at least somewhat".

\section{Discussion}

Engaging patients and their families as active participants and collaborators in care is critical for achieving patient centered health care in the U.S., improving the patient experience of care, improving outcomes and reducing the per capita costs of health care [49]. Among

Table 3 Change in Scores between Baseline and Final Follow-up

\begin{tabular}{llll}
\hline & PDA & Usual Care & $p$-value \\
& $n=114$ & $n=111$ & \\
& $($ Mean $\triangle$, SD) & $($ Mean $\triangle$, SD) & \\
\hline Knowledge (\% correct) & $35.0 \%(22.3)$ & $9.9 \%(22.2)$ & $<.0001$ \\
Knowledge confidence & $11.0(12.8)$ & $1.6(8.9)$ & $<.0001$ \\
Decision Self-Efficacy scale & $3.7(16.7)$ & $-3.9(19.2)$ & 0.0018 \\
Decisional Conflict Score (total) $)^{b}$ & $-22.2(20.6)$ & $-7.5(16.6)$ & $<.0001$ \\
$\quad$ Uncertainty subscale & $-21.3(27.3)$ & $-9.5(23.5)$ & 0.0006 \\
$\quad$ Informed subscale & $-29.9(26.5)$ & $-8.4(27.4)$ & $<.0001$ \\
$\quad$ Values Clarity subscale & $-27.1(24.4)$ & $-8.9(22.1)$ & $<.0001$ \\
$\quad$ Support subscale & $-19.1(20.7)$ & $-5.8(17.1)$ & $<.0001$ \\
$\quad$ Effective Decision subscale & $-15.7(22.1)$ & $-5.4(18.6)$ & $<.0001$ \\
\hline${ }^{a t-t e s t}$ & & & \\
${ }^{b}$ Lower is considered better & & &
\end{tabular}

people with diabetes, the Diabetes Attitudes, Wishes and Needs (DAWN2) study recently indicated that engagement and participation by people with diabetes is lacking, but is a high priority for both patients and health professionals $[50,51]$. In addition, patient engagement is particularly important in the management of high-risk diabetes patients [52]. However, shared decision-making that provides the amount and depth of information targeted to what is important to patients in support of meaningful patient-provider collaboration is time consuming and contributes to delays in the advancement and intensfication of treatment [53, 54]. The development and use of health information using technology platforms, such as this PDA, is effective, acceptable to patients, and has the potential to provide the knowledge and confidence patients need to more fully participate in shared decision-making and become true partners in self-management and their future health. To support communication and engagement in decision making, we developed and tested the Diabetes Decision Aid for T2DM. This decision aid is the first for T2DM widely available in an electronic format that not only provides targeted information on medication choices, but also facilitates users' understanding of personal values related to important aspects of the medication decision through a values a clarification exercise, including each option's risks and benefits. Values clarification help patients understand and recognize if treatment decision are consistent with the priorities identified in the exercise. Helping patients make explicit value assumptions may support decision-making processes with fuller understanding and buy in, which is integral to one's self- 
Table 4 Adjusted analyses - primary and secondary outcomes. Generalized linear model regression model -primary and secondary outcomes

\begin{tabular}{|c|c|c|c|c|c|c|c|c|}
\hline & \multicolumn{2}{|c|}{ Percent Correct Response $\Delta$} & \multicolumn{2}{|c|}{ Knowledge Confidence $\Delta$} & \multicolumn{2}{|c|}{ Decisional Conflict $\Delta$} & \multicolumn{2}{|c|}{ Decisional Self Efficacy $\Delta$} \\
\hline & Coefficient (SE) & $P$ value, $5 \% \mathrm{Cl}$ & Coefficient (SE) & $\mathrm{P}$ value, $95 \% \mathrm{Cl}$ & Coefficient (SE) & $P$ value, $95 \% \mathrm{Cl}$ & Coefficient (SE) & $P$ value, $95 \% \mathrm{Cl}$ \\
\hline PDA Group & $26.60(3.23)$ & $<.0001(20.23,32.96)$ & $10.13(1.54)$ & $<.0001(7.10,13.17)$ & $-16.25(2.57)$ & $<.0001(-1.33,-11.18)$ & $7.44(2.56)$ & $0.004(2.39,12.49)$ \\
\hline Male & $6.74(3.26)$ & $0.040(0.31,13.18)$ & $1.27(1.55)$ & $0.414(-1.79,4.34)$ & $1.78(2.60)$ & $0.494(-3.34,6.91)$ & $0.67(2.59)$ & $0.796(-4.43,5.77)$ \\
\hline Non-adherence with PDA & $-0.50(6.77)$ & $0.941(-13.85,12.85)$ & $-5.57(3.23)$ & $0.086(-11.93,0.79)$ & $8.02(5.39)$ & $0.139(-2.62,18.65)$ & $-6.65(5.37)$ & $0.217(-17.24,3.94)$ \\
\hline Education - Grade School* & $-4.34(14.27)$ & $0.762(-32.48,23.80)$ & $-6.92(6.80)$ & $0.310(-20.33,6.49)$ & $-28.07(11.37)$ & $0.014(-50.49,-5.65)$ & $20.28(11.32)$ & $0.075(-2.04,42.60)$ \\
\hline Education - Some High School* & $-5.31(8.07)$ & $0.512(-21.22,10.61)$ & $-6.14(3.85)$ & $0.112(-13.72,1.44)$ & $7.39(6.43)$ & $0.252(-5.29,20.06)$ & $-3.72(6.40)$ & $0.562(-16.34,8.90)$ \\
\hline Education - High School Graduate* & $1.55(5.61)$ & $0.782(-9.51,12.62)$ & $-5.88(2.55)$ & $0.002(-13.78,3.24)$ & $3.81(4.47)$ & $0.396(-5.01,12.62)$ & $-2.90(4.45)$ & $0.516(-11.68,5.88)$ \\
\hline Education - Some College* & $4.98(5.36)$ & $0.354(-5.58,15.54)$ & $-7.31(2.67)$ & $0.022(-10.92,-0.85)$ & $-1.56(4.27)$ & $0.716(-9.97,6.85)$ & $-6.05(4.25)$ & $0.156(-14.42,2.33)$ \\
\hline Education - College Graduate* & $2.67(5.61)$ & $0.635(-8.40,13.73)$ & $3.78(2.85)$ & $0.007(-12.58,-2.04)$ & $2.11(4.47)$ & $0.638(-6.71,10.92)$ & $-5.57(4.45)$ & $0.212(-14.35,3.20)$ \\
\hline $\begin{array}{l}\text { Stage of Decision-makingt - Have not } \\
\text { begun to think about choice }\end{array}$ & $-2.03(5.99)$ & $0.735(-13.83,9.77)$ & $-7.31(2.67)$ & $0.186(-1.84,9.41)$ & $-12.86(4.77)$ & $0.008(-22.26,-3.46)$ & $3.90(4.75)$ & $0.413(-5.46,13.26)$ \\
\hline $\begin{array}{l}\text { Stage of Decision-making }{ }^{\dagger} \text { - Have not } \\
\text { begun to think about choice, } \\
\text { but interested }\end{array}$ & $1.36(6.15)$ & $0.825(-10.76,13.49)$ & $6.71(2.93)$ & $0.023(0.93,12.49)$ & $-11.78(4.90)$ & $0.017(-21.44,-2.12)$ & $6.71(4.88)$ & $0.171(-2.91,16.33)$ \\
\hline $\begin{array}{l}\text { Stage of Decision-making }{ }^{\dagger} \text { - Considering } \\
\text { options/close to selecting }\end{array}$ & $1.05(6.40)$ & $0.870(-11.57,13.67)$ & $5.75(3.05)$ & $0.061(-0.27,11.76)$ & $-14.64(5.10)$ & $0.005(-24.7,-4.58)$ & $2.34(5.08)$ & $0.645(-7.67,12.35)$ \\
\hline $\begin{array}{l}\text { Stage of Decision-making }{ }^{\dagger} \text {-already } \\
\text { made decision, but willing to consider }\end{array}$ & $0.54(6.83)$ & $0.938(-12.94,14.01)$ & $5.68(3.26)$ & $0.083(-0.74,12.10)$ & $-7.37(5.44)$ & $0.177(-18.11,3.36)$ & $6.37(5.42)$ & $0.241(-4.32,17.06)$ \\
\hline Previous yeast or UTI History & $-0.82(4.62)$ & $0.860(-9.92,8.28)$ & $1.80(2.20)$ & $0.414(-2.54,6.14)$ & $-3.24(3.68)$ & $0.379(-10.49,4.01)$ & $-0.42(3.66)$ & $0.909(-7.64,6.80)$ \\
\hline Values -finding lowest cost option & $-0.14(0.64)$ & $0.825(-1.40,1.12)$ & $-0.06(0.30)$ & $0.840\{-0.66,0.54)$ & $-0.20(0.51)$ & $0.698(-1.20,0.81)$ & $0.67\{0.51)$ & $0.190(-0.33,1.67)$ \\
\hline Race- Black/African American \pm & $-0.24(3.81)$ & $0.950(-7.75,7.27)$ & $-0.96(1.82)$ & $0.598(-4.54,2.62)$ & $-0.18(3.04)$ & $0.954(-6.16,5.81)$ & $-0.66(3.02)$ & $0.828(-6.62,5.30)$ \\
\hline Race - Hispanic \pm & $-3.35(4.84)$ & $0.489(12.89,6.19)$ & $-1.86(2.30)$ & $0.422(-6.40,2.69)$ & $2.78(3.85)$ & $0.471(-4.81,10.38)$ & $-6.27(3.84)$ & $0.104(-13.84,1.29)$ \\
\hline Race - Other \pm & $-2.27(4.95)$ & $0.647(-12.03,7.48)$ & $2.06(2.36)$ & $0.384(-2.59,6.70)$ & $5.37(3.94)$ & $0.175(-2.41,13.14)$ & $1.23(3.92)$ & $0.755(-6.51,8.97)$ \\
\hline Intercept & $5.91(8.67)$ & $0.49(11.18,23.01)$ & $2.75(4.13)$ & $0.507(-5.40,10.90)$ & $3.10(6.91)$ & $0.654(-10.52,16.72)$ & $-8.56(6.88)$ & $0.215(-2212,5.00)$ \\
\hline
\end{tabular}

*Reference condition: Education - Graduate School

${ }^{\dagger}$ Reference condition: Stage of Decision-making - Decided, unlikely to change mind \pm Reference condition: Caucasian 
confidence or belief in the ability to make decisions (decision self-efficacy) $[55,56]$.

PDA use was associated with substantial and significant improvements in knowledge about T2DM medication, decisional self-efficacy, and decisional conflict in a large socio-demographically diverse sample. We discovered several apparent contradictions. Even though all subjects had been advised by their provider to consider additional antihyperglycemic medication, more than half of the subjects in both groups reported that they had not begun to consider their treatment options. Most (70 \%) participants preferred an active role in decisionmaking, yet few had adequate knowledge to make a good decision. This suggests a substantial unmet need to both activate patients to engage in decision-making and to help them improve their knowledge about T2DM and its treatment.

\section{Knowledge}

The gains in knowledge that we observed in our diverse national sample (approximately 23 points on a normalized scale) are consistent with findings from other PDAs attempting to improve knowledge about diabetes antihyperglycemic medication options in different settings. Branda et al. reported knowledge gains of 23.5 [95 \% CI 9.7, 37.3] among a largely Caucasian sample of T2DM patients in rural Minnesota [35]. In this study, the patients receiving the Diabetes Choice PDA achieved a final correct score of $57 \%$ and the control group achieved a correct score of $33 \%$, similar to our findings of 55.5 and $32.5 \%$ correct, respectively. Both studies demonstrated that although gains in knowledge and knowledge confidence were substantial in the PDA groups. The Statin Choice Trial [28], conducted among patients with diabetes exploring adding statins to their medication regimens, found that their PDA improved knowledge of medication risks and benefits by approximately 27 points in a highly educated Caucasian population in Minnesota, administered during a face-to-face consultation. The PANDAs PDA [34] included predominantly well-educated Caucasians in the UK and reported mixed results for knowledge when two questions were asked to assess understanding of medication effectiveness and risks. Our study provides further evidence of a substantial gap in knowledge about T2DM and its treatment in an educationally and racially/ ethnically diverse national sample. Our findings support the need for better targeted patient education about T2DM and antihyperglycemic treatments to help patients prepare for shared decision-making [52].

\section{Decsional self efficacy}

Improvements in decisional self-efficacy were found in the PDA group. This may have been due to the positive relationship between self-reported efficacy expectations and knowledge acquisition [15, 57]. An unexpected outcome was a decrease in decisional self-efficacy in the UC group. When people are confronted with a challenging task, some may become less sure of their efficacy [58]. Baseline decisional self-efficacy was measured prior to administering a detailed knowledge test and a questionnaire addressing the many factors that must be considered when selecting medication (e.g., minimizing the risk of hypoglycemia, adverse events). Subjects may have overestimated their self-efficacy at baseline [53], and UC self-efficacy may have been negatively impacted upon recognizing how little subjects actually knew about their medication options and how many factors needed to be considered. To our knowledge, this study is the first to observe a decrease in self-efficacy in the control arm of studies of treatment-focused patient decision aids. Of 115 studies in the current Cochrane systematic review, 8 measured the effect of decision aids on confidence when comparing decision aid use to usual care, 3 of which used decisional self-efficacy as that measure of confidence. None of these studies measured outcomes related to a decision aid for diabetes and only one measured change in DSES from baseline. Atterbern et al. [59] randomized subjects to a video PDA vs written materials for information regarding bariatric surgery. No decreases in self-efficacy were observed in the control group receiving the pamphlet, and no significant differences in DSES change from baseline were observed in the treatment group versus the control group.

\section{Decisional conflict}

The PDA significantly lowered overall decisional conflict and each of its subscales. Mean DCS scores at final follow-up were $<25$, the threshold below that equates to making an effective decision [43]. Some of these findings were unexpected because the PDA did not explicitly target some of the domains measured by the subscale, such as feeling more supported in their decision-making. Only six of 15 treatment-related PDAs in the most recent Cochrane review reported significant findings for this subscale [26]. These improvements in decisional conflict subscales may reflect unique features of our PDA, such as tailoring information according to the patients' preferences (e.g., expected degree of glycemic control, and durability of control; impact on weight; risk of hypoglycemia; costs). This type of tailoring might make patients feel more supported in their choice. The PDA also includes interactive values clarification exercises that help patients weigh relative the importance of each of these values, which is different than the more common static printed or videotaped PDA material. These exercises may explain our positive impact on the values clarity subscale. 


\section{Limitations}

Our study has several limitations. First, our blinding of referring clinicians prevented us from engaging providers in shared decision-making training, which has been shown to augment the effect of PDAs [60]. Thus our findings may underestimate the impact of our PDA where provider shared decision-making training is included. On the other hand, subjects were not blinded to treatment assignment, and this may have impacted results due to expectations raised regarding PDA participation benefits. Also, because viewing the PDA and assessments were conducted over the internet, results may not be generalizable to a lessinternet experienced audience, or to an audience with socioeconomic barriers to internet access. Our choice of a comparator, usual care, is not an ideal comparison for PDA assessments. An alternative may have been to randomize control subjects to a time-matched subject information page. This would help assure that differences between groups was related to PDA content, and not the system of support inherent and facilitated by the PDA delivery system. Our sample size was relatively small, thus larger studies are warranted. Although we enrolled a diverse group of subjects, we did not directly measure literacy or numeracy but instead used educational level as a proxy.

\section{Conclusions}

This study provides insights into the impact of a novel interactive PDA for subjects with T2DM for whom first line treatment with metformin is no longer effective. The PDA helps patients acquire essential knowledge to make informed decisions according to their personal values and preferences. It substantially and significantly improved knowledge, decisional self-efficacy, and decisional conflict among a diverse group of patients with T2DM. We developed a Spanish translation of the PDA for a Latino/Hispanic audience, but have not yet tested it. It would be important to understand its impact in Spanish-speaking populations. The PDA is currently available and being deployed in a variety of US health systems (www.diabetesdecisionaid.com. Future studies should assess the impact of PDA on medication choice, medication adherence, patient-provider communication, glycemic control, other clinical outcomes, and costs.

\section{Ethics apprproval and consent to participate}

All study procedures, including informed consent were approved by the New England IRB (\#14-104).

\section{Consent for publication}

Not applicable.

\section{Availability of data and materials}

Data and materials are available upon written request to the corresponding author.

\section{Additional file}

Additional file 1: T2DM Medication Knowledge Questions. (DOCX $16 \mathrm{~kb}$ )

\begin{abstract}
Abbreviations
DAWN2: Diabetes attitudes, wishes and needs (DAWN2) STUDY;

DCS: Decisional conflict scale; DPP-4: Dipeptidyl peptidase-4; DSES: Decisional self efficacy scale; GLM: Generalized Linear Model; GLP-1: Glucagon-like peptide-1 (GLP-1S) agonists; ITT: Intent to treat; MCMC: Markov-Chain-MonteCarlo; MO: Missouri; PDA: Patient decision aid; SAS: Statistics analytics system; SD: Standard deviation; SGLT-2: Sodium-glucose co-transporter 2 inhibitors; T2DM: Type 2 diabetes mellitus; TZD: Thiazolidinediones; UC: Usual care; US: United States.
\end{abstract}

\section{Competing interests}

R Bailey is an employee of Janssen Scientific Affairs, LLC and shareholder of Johnson and Johnson. Michael Pfeifer is an employee and shareholder of Johnson and Johnson. Alicia Shillington is an employee and shareholder of EPI-Q Inc. Qing Harshaw is an employee of EPI-Q Inc. Jeffery VanWingen is in private practice and received compensation from Janssen Scientific Affairs for enrollment of subjects into this investigation. Nananda Col is a consultant to Janssen Scientific Affairs. Martha Funnell has served on advisory boards for Eli Lilly, Bristol-Myers Squibb, AstraZeneca Diabetes, Novo Nordisk, Omada Health, Sanofi US, and is a consultant to Janssen Scientific Affairs.

\section{Authors' contributions}

All authors had access to the data and played a role in writing this manuscript. ACS and RAB conceived and designed this study; NC contributed to study design, ACS and JWW contributed to the collection of study data; $\mathrm{QH}$ and ACS analyzed data for the study. All authors (RAB, MP, ACS, QH, MMF, JWW, NC) contributed interpretation of study data, reviewed and critically revised manuscript drafts and approved the manuscript for publication.

\section{Funding}

The trial and manuscript submission was funded by Janssen Scientific Affairs, LLC.

\section{Author details}

'Janssen Scientific Affairs, LLC, Raritan, NJ, USA. ${ }^{2}$ EPI-Q Inc, 1315 W 22nd Street, Suite 410, Oakbrook, IL 60523, USA. ${ }^{3}$ Department of Learning Health Sciences, University of Michigan Medical School, Ann Arbor, MI, USA. ${ }^{4}$ Family Medicine Specialists, Grand Rapids, MI, USA. ${ }^{5}$ Five Islands Consulting, Georgetown, ME, USA.

Received: 27 October 2015 Accepted: 7 January 2016

Published online: 14 January 2016

\section{References}

1. Sarkar U, Fisher L, Schillinger D. Is self-efficacy associated with diabetes self-management across race/ethnicity and health literacy? Diabetes Care. 2006;29(4):823-9.

2. Tunrer R, on behalf of the UKPDS Study Group. Intensive blood-glucose control with sulphonylureas or insulin compared with conventional treatment and risk of complications in patients with type 2 diabetes (UKPDS 33). Lancet. 1999;354:602.

3. Shamoon $\mathrm{H}$, Duffy $\mathrm{H}$, Fleischer $\mathrm{N}$, Engel $\mathrm{S}$, et al. The effect of intensive treatment of diabetes on the development and progression of long-term complications in insulin-dependent diabetes mellitus. The diabetes control and complications trial research group. N Engl J Med. 1993;329(14):977-86.

4. Garber A, Abrahamson M, Barzilay J, Blonde L, Bloomgarden Z, Bush M, et al. AACE/ACE Comprehensive diabetes management algorithm 2015. Endo Pract. 2015;21:438-47.

5. Williams M, Baker D, Parker R, Nurss J. Relationship of functional health literacy to patients' knowledge of their chronic disease - A study of patients with hypertension and diabetes. Arch of Int Med. 1998;158:166-72.

6. Nam S, Chesla C, Stotts NA, Kroon L, Janson SL. Barriers to diabetes management: patient and provider factors. Diabetes Res Clin Pract. 2011;93:1-9. 
7. Fisher $L$, Hessler D, Masharani U. Impact of baseline patient characteristics on interventions to reduce diabetes distress: the role of personal conscientiousness and diabetes self-efficacy. Diabet Med. 2014;31(6):739-46.

8. Martin-Harris B, Gebregziabher M, Walker Rebekah J, Egede LE, et al. Understanding the influence of psychological and socioeconomic factors diabetes self-care using structured equation modeling. Patient Educ Couns. 2015;98(1):34-40.

9. Schwarzer R. Modeling health behavior change: how to predict and modify the adoption and maintenance of health behaviors. J Appl Psychol. 2008; 57(1):1-29.

10. O'Connor AM. Validation of a decisional conflict scale. Med Decis Making. 1995;15(1):25-30.

11. O'Connor AM, Rostom A, Fiset V, Tetroe J, Entwistle V, Llewellyn-Thomas H, et al. Decision aids for patients facing health treatment or screening decisions: systematic review. BMJ. 1999;319(7212):731-4.

12. King DK, Glasgow RE, Toobert DJ, Strycker LA, Estabrooks PA, Osuna D, et al. Self-efficacy problem solving, and social-environmental support are associated with diabetes self-management behaviors. Diabetes Care. 2010; 33(4):751-3.

13. Gonzalez JS, Shreck P, Psaros C, Safrin SA. Distress and type 2 diabetes-treatment adherence: a mediating role for perceived control. Health Psychol. 2015;34(5): 505-13.

14. Adam J, Folds L. Depression, self-efficacy, and adherence in patients with type 2 diabetes. J Nur Pract. 2014;10(9):42-8.

15. Berikai P, Meyer PM, Kazlauskaite R, Savoy B, Kozik K, Fogelfeld L. Gain in patients' knowledge of diabetes management targets is associated with better glycemic control. Diabetes Care. 2007;20(6)1587-1589.

16. Sousa VD, Zauszniewski JA, Musil CM, Price Lea PJ, Davis SA. Relationships among selfcare agency, self-efficacy, self-care, and glycemic control. Res Theory Nurs Pract. 2005;19(3):217-30

17. McPherson ML. Association between diabetes patients' knowledge about medications and their blood glucose control. Res SocialAdmin Pharm. 2008; 4(1):37-45.

18. American Diabetes Association. Standards of medical care in diabetes. Diabtes Care. 2014;37 Suppl 1:S14-80.

19. Moreno G, Mangione CM, Kimbro L, Vaisberg E. Guidelines abstracted from the American Geriatrics Society Guidelines for Improving the Care of Older Adults with Diabetes Mellitus: 2013 update. J Am Geriatr Soc. 2013;61(11): 2020-6.

20. Woolf SH, Chan ECY, Harris R, Sheridan SL, Braddock 3rd CH, Kaplan RM, et al. Promoting informed choice: transforming health care to dispense knowledge for decision making. Ann Intern Med. 2005;143(4):293-300.

21. Barry MJ, Edgman-Levitan S. Shared decision making-pinnacle of patientcentered care. NEJM. 2012;366(9):780-1.

22. Braddock 3rd CH, Edwards KA, Hasenberg NM, Laidley TL, Levinson W. Informed decision making in outpatient practice: time to get back to basics. JAMA. 1999;282(24):2313-20.

23. Lipska KJ, Ross JS, Miao Y, Shah ND, Lee SJ, Steinman MA. Potential overtreatment of diabetes mellitus in older adults with tight glycemic control. JAMA. 2015;175(3):356-62.

24. Tseng CL, Soroka O, Maney M, Aron DC, Pogach LM. Assessing potential glycemic overtreatment in persons at hypoglycemic risk. JAMA Intern Med. 2014;174:259-68.

25. Institute of Medicine. Crossing the quality chasm. https://iom.nationalacademies. org/ /media/Files/Report\%20Files/2001/Crossing-the-Quality-Chasm/ Quality\%20Chasm\%202001\%20\%20report\%20brief.pdf. Accessed October 7, 2015.

26. Legare F, Ratte S, Gravel K. Barriers and facilitators to implementing shared decision-making in clinical practice: update of a systematic review of health professionals' perceptions. Pat Ed and Couns. 2008;73(3):526-35.

27. Stacey D, Legare F, Col NF, et al. Decision aids for people facing health treatment or screening decisions. Cochrane Database Syst Rev. 2014;1:1-332.

28. Nannenga MR, Montori VM, Weymiller AJ, Smith SA, Christianson TJ, Bryant SC, et al. A treatment decision aid may increase patient trust in the diabetes specialist: the Statin choice randomized trial. Health Expect. 2009; 12:38-44

29. Weymiller AJ, Montori VM, Jones LA, Gafni A, Guyatt GH, Bryant SC, et al. Helping patients with type 2 diabetes mellitus make treatment decisions: statin choice randomized trial. Arch Intern Med. 2007;167:1076-82.

30. Jones LA, Weymiller AJ, Shah N, Bryant SC, Christianson TJ, Guyatt GH, et al. Should clinicians deliver decision aids? Further exploration of the statin choice randomized trial results. Med Decis Making. 2009;29:468-74.
31. Mann DM, Ponieman D, Montori VM, Arciniega J, McGinn T. The Statin Choice decision aid in primary care: a randomized trial. Patient Educ Couns. 2010;80:138-40.

32. Corser W, Holmes-Rovner M, Lein C, Gossain V. A shared decision-making primary care intervention for type 2 diabetes. Diabetes Educ. 2007;33(4):700-8.

33. Denig P, Schuling J, Haaijer-Ruskamp F, Voorham J. Effects of a patient oriented decision aid for prioritising treatment goals in diabetes: pragmatic randomised controlled trial. BMJ. 2014;349:g5651.

34. Edwards A, Thomas R, Williams R, Ellner AL, Brown P, Elwin G. Presenting risk information to people with diabetes: evaluating effects and preferences for different formats by a web-based randomised controlled trial. Pat Edu Couns. 2006;63:336-49.

35. Mathers N, Ng CJ, Campbell MJ, Colwell B, Brown I, Bradley A. Clinical effectiveness of a patient decision aid to improve decision quality and glycaemic control in people with diabetes making treatment choices: a cluster randomised controlled trial (PANDAs) in general practice. BMJ OPEN. 2012;2(6):e001469.

36. Branda ME, LeBlanc A, Shah ND, Tiedje K, Ruud K, Van Houten H, et al. Shared decision making for patients with type 2 diabetes: a randomized trial in primary care. BMC Heal Serv Res. 2013;13:30113.

37. Anderson RM, Funnell MM, Butler PM, Arnold MS, Fitzgerald JT, Feste CC. Patient empowerment: results of a randomized controlled trial. Diabetes Care. 1995;18(7):943-9.

38. Detry MA, Lewis RJ. The intention-to-treat principle: how to assess the true effect of choosing a medical treatment. JAMA. 2014;312(1):85-6.

39. Montori VM, Guyatt GH. The intention-to-treat principle. Can Med Assoc J. 2001;165(10):1339-41.

40. Shillington AC, Col N, Bailey RA, Jewell MA. Development of a patient decision aid for type 2 diabetes mellitus for patients not achieving glycemic control on metformin alone. Patient Pref Adherence. 2015;9:609-17.

41. Fagerlin A, Pignone M, Abhyankar P, Col N, Feldman-Stewart D, Gavaruzzi T, et al. Clarifying values: an updated review. BMC Med Inform Decis Mak. 2013;13 Suppl 2:S8.

42. International Patient Decision Aids Standards Collaboration. http://ipdas.ohri. ca/ Accessed Oct 1, 2015.

43. O'Connor A. User manual - stage of decision making. https://decisionaid. ohri.ca/docs/develop/User_Manuals/UM_Stage_Decision_Making.pdf. Accessed Oct 1, 2015

44. Degner LF, Sloan JA, Venkatesh P. The control preferences scale. Can J Nurs Res. 1997;29(3):21-43.

45. O'Connor A, Bunn $\mathrm{H}$. Validation of client decision-making instruments in the context of psychiatry. Can J Nurs Res. 1996;28(3):13-27.

46. O'Connor A. User manual - decisional conflict scale. https://decisionaid.ohri.ca/ docs/develop/User_Manuals/UM_Decision_SelfEfficacy.pdf. Accessed Oct 1, 2015.

47. https://decisionaid.ohri.ca/docs/develop/User_Manuals/UM_Decisional_ Conflict.pdf. Accessed Oct 1, 2015

48. Horton NJ. Multiple Imputation in practice. The Am Stat. 2001;55(3):244-54.

49. Paget L, Salzberg C, Scholle SH. Building a strategy to leverage health information technology to support patient and family engagement. http://www.ncqa.org/HEDISQualityMeasurement/Research/ BuildingaStrategytoLeverageHealthInformationTechnology.aspx. Accessed October 10, 2015

50. on behalf of he DAWN2 Study Group, Nicolucci A, Burns KK, Holt RIG, Comaschi M, Hermanns N, et al. Diabetes attitudes, wishes and needs second study (DAWN2 ${ }^{\mathrm{TM}}$ ): Cross-national benchmarking of diabetes-related psychosocial outcomes for people with diabetes. Diabet Med. 2013;30:767-77.

51. Holt RI, Nicolucci A, Kovacs Burns K, Escalante M, Forbes A, Hermanns N, et al. DAWN2 Study Group. Diabetes attitudes, wishes and needs second study (DAWN2 ${ }^{\mathrm{TM}}$ ): cross-national comparisons on barriers and resources for optimal care-healthcare professional perspective. Diabet Med. 2013;30:789-98.

52. Powers MA, Bardsley J, Cypress M, Duker P, Funnell MM, Fischl AH, et al. Diabetes self-management education and support in type 2 diabetes: a joint position statement of the American Diabetes Association, the American Association of Diabetes Educators and the Academy of Nutrition and Dietetics. Diabetes Care. 2015;38:1372-82.

53. Khunti K, Wolden ML, Thorsted BL, Andersen M, Davies JF. Clinical inertia in people with type 2 diabetes: a retrospective cohort study of more than 80,000 people. Diabetes Care. 2013;36:3411-7.

54. Ratanawongsa N, Crosson JC, Schillinger D, Karter AJ, Saha CK, Marrero DG. Getting under the skin of clinical inertia in insulin initiation: the translating research into action for diabetes (TRIAD) insulin starts project. Diabetes Educ. 2012;38:94-100. 
55. Charles C, Gafni A, Whelan T, O'brien M. Treatment decision aids: conceptual issues and future directions. Health Exp. 2005;8(2):114-25.

56. O'Connor A, Wells G, Tugwell P, Laupacis A, Elmslie T, Drake E. The effect of an explicit values clarification exercise in a women's decision aid regarding postmenopausal hormone therapy. Health Exp. 1999;2:21-32.

57. Bandura A. Self-efficacy: toward a unifying theory of behavioral change. Psy Rev. 1977;84(2):191-215.

58. Bandura A. Negative self-efficacy and goal effects revisited. J of Appl Psychol. 2003;88(1):87-99.

59. Atterburn DE, Westbrook EO, Bogart TA, Sepucha KR. Randomized trial of a video-based patient decision aid for bariatric surgery. Obesity. 2011;19:1669-75,

60. Legare F, Labrecque M, LeBlanc A, Njoya M, Laurier C, Côté L, et al. Training family physicians in shared decision making for the use of antibiotics for acute respiratory infections: a pilot clustered randomized controlled trial. Health Exp. 2010;14 Suppl 1:96-110.

Submit your next manuscript to BioMed Central and we will help you at every step:

- We accept pre-submission inquiries

- Our selector tool helps you to find the most relevant journal

- We provide round the clock customer support

- Convenient online submission

- Thorough peer review

- Inclusion in PubMed and all major indexing services

- Maximum visibility for your research

Submit your manuscript at www.biomedcentral.com/submit
C) Biomed Central 